\title{
Usefulness of Abdominal Massage and ST-36 (Zusanli) Acupressure in Improving The Appetite of COVID-19 Patients Treated in a Makeshift Hospital: A Randomized Controlled Trial
}

\section{Lei Wang}

Jiangsu Province Hospital and Nanjing Medical University First Affiliated Hospital

Jifang Peng

Jiangsu Province Hospital and Nanjing Medical University First Affiliated Hospital

Hairong Liu

Jiangsu Province Hospital and Nanjing Medical University First Affiliated Hospital

Yan Jia

Jiangsu Province Hospital and Nanjing Medical University First Affiliated Hospital

Cui Yao

Jiangsu Province Hospital and Nanjing Medical University First Affiliated Hospital

Yufan Lin

Jiangsu Province Hospital and Nanjing Medical University First Affiliated Hospital

Hui Yua

Jiangsu Province Hospital and Nanjing Medical University First Affiliated Hospital

Fang Li ( $\square$ lifangcf@163.com)

Jiangsu Province Hospital and Nanjing Medical University First Affiliated Hospital

\section{Research Article}

Keywords: COVID-19, makeshift hospital, appetite, abdominal massage, ST-36 acupressure

Posted Date: July 12th, 2021

DOl: https://doi.org/10.21203/rs.3.rs-642536/v1

License: (c) (1) This work is licensed under a Creative Commons Attribution 4.0 International License. Read Full License 


\section{Abstract}

Objectives: To investigate the appetite of COVID-19 patients treated in a makeshift hospital and to evaluate the effectiveness of abdominal massage and ST-36 (Zusanli) acupressure in improving their appetite.

Design: Randomized controlled trial.

Methods: This study was performed between February 15 and March 2, 2020, in a sports centre makeshift hospital in Wuhan, China. The appetite of 110 COVID-19 patients was assessed using a mobile app-based Simplified Nutritional Appetite Questionnaire (SNAQ). The experimental and control groups, 55 patients each, were randomized using a prepared randomization checklist. Through video guidance and on-the-spot instruction, patients in the experimental group mastered the methods of abdominal massage and ST-36 (Zusanli) acupressure and implemented the intervention for 7 days by themselves. The appetite of the patients was assessed again after intervention.

Results: In a total of 110 participants, 43 (39.1\%) patients suffered from a poor appetite. A positive correlation between poor appetite and older age as well as fever was observed $(p<0.05)$. In the experimental group, the SNAQ scores were significantly increased $(14.07 \pm 2.46$ vs $15.55 \pm 1.77, p=0.0004)$, and the proportion of patients with a poor appetite was markedly reduced after the intervention ( $41.8 \% \mathrm{vs}$ $10.9 \%, p=0.0002)$. Compared with the control group, the rate of poor appetite in the experimental group was significantly decreased postintervention $(27.3 \%$ vs $10.9 \%, p=0.029)$.

Conclusion: In COVID-19 patients, a poor appetite may be correlated with older age and fever. Abdominal massage and ST-36 (Zusanli) acupressure are applicable and effective in improving the appetite of COVID-19 patients during isolation treatment.

Trial registration: Chinese clinical trial registry (ChiCTR2000033261), the trial was registered retrospectively.

\section{Introduction}

During an outbreak of pneumonia in Wuhan, Hubei Province, China, in late December 2019, a novel coronavirus named severe acute respiratory syndrome coronavirus-2 (SARS-CoV-2) was identified as the causative pathogen ${ }^{1}$. Since the virus possesses powerful transmissibility, the number of confirmed cases of 2019 novel coronavirus disease (COVID-19) has increased rapidly in China and around the world. COVID-19 has been declared a global pandemic by the $\mathrm{WHO}^{2}$.

The most important strategy for controlling the COVID-19 pandemic is breaking the chain of transmission, which means finding, isolating, testing and treating every patient as early as possible. As the first most seriously affected country, China implemented a series of effective actions to prevent and treat the pandemic. The rates of confirmed cases showed a downward trend, and cured cases increased 
rapidly. Makeshift hospitals that were temporarily set up in large venues received the majority of mild individuals for isolation treatment, leading to a considerable reduction in their stress during COVID-19 control.

It is generally believed that nutritional support is one of the indispensable therapies for infectious diseases. Emerging evidence has suggested nutritional status as a relevant factor influencing the outcome of COVID-19 patients ${ }^{3}$. Because of limited movement areas and psychological anxiety, patients in makeshift hospitals may have gastrointestinal symptoms such as bloating, loss of appetite, constipation, and diarrhoea. These problems may impede nutrients absorption. Furthermore, SARS-CoV-2 can cause gastrointestinal symptoms leading to malnutrition ${ }^{4}$. The prevalence of malnutrition in elderly patients with COVID-19 is high, and nutritional support should be strengthened during treatment ${ }^{5}$. Most COVID-19 patients treated in makeshift hospitals were mild type, and their dietary status was closely related to their nutritional status. Thus, the appetite of these patients deserves our attention.

Abdominal massage and ST-36 (Zusanli) acupressure have been reported to have beneficial effects on appetite improvement and gastrointestinal function in various diseases ${ }^{6-10}$. However, in the setting of makeshift hospitals, the effect of abdominal massage and ST-36 (Zusanli) acupressure on improving COVID-19 patients' gastrointestinal function remains unknown.

In this study, we evaluated the appetite of 110 mild COVID-19 confirmed patients in a sports centre makeshift hospital using a mobile phone app-based questionnaire survey. The enrolled patients were randomly divided into the experimental and control groups. Abdominal massage and ST-36 (Zusanli) acupressure were applied in the experimental group, and the effect was assessed 7 days after the intervention. The aim of this study was to provide some experience for caring for mild COVID-19 patients during isolation treatment.

\section{Methods}

\subsection{Design}

A randomized controlled trial was conducted in the Sports Center makeshift hospital in Wuhan between February 15 and March 2, 2020. This study was based on the CONSORT statement for randomized controlled trials.

\subsection{Participants}

A total of 120 patients hospitalized in Sports Center Makeshift Hospital in Wuhan between February 15 and March 2, 2020 were recruited after obtaining written informed consent. The inclusion criteria were as follows: (1) patients with a confirmed diagnosis from a positive test for COVID-19 RT-PCR who were aged more than 18 years old; (2) patients who were classified as mild or common clinical types according to the "Diagnosis and Treatment Protocol for Novel Coronavirus Infection-Induced Pneumonia version 5 (trial)"; and (3) patients who were able to skilfully use the mobile phone app. The exclusion criteria 
included patients who had severe cognitive impairment, digestive disease and no phone equipment. Four patients were excluded due to a pre-existing digestive disease. A total of 116 patients were randomized into the experimental group $(n=58)$ or the control group $(n=58)$ by a computer-generated randomization list. Two patients in the experimental group and three patients in the control group were excluded due to leaving the hospital. One patient in the experimental group quit the study due to intolerance of the intervention. Ultimately, 110 patients were enrolled for the final analysis, with 55 in the experimental group and 55 in the control group.

\subsection{Ethical considerations}

This study was approved by the Ethics Committee of the First Affiliated Hospital of Nanjing Medical University (approval number: 2020-SR-149) and was registered in the Chinese clinical trial registry (ChiCTR2000033261).

\subsection{Data collection}

A self-designed questionnaire was used to collect demographic data, including sex, age, educational background, marital status and fever.

The Simplified Nutritional Appetite Questionnaire (SNAQ), which was adopted in this study to evaluate the patients' appetite, includes 4 questions as follows: 1. My appetite is (A. Very poor/B. Poor/C. Average/D. Good/E. Very good); 2 . When I eat (A. I feel full after eating only a few mouthfuls/B. I feel full after eating about a third of a meal/C. I feel full after eating over half of a meal/D. I feel full after eating most of a meal/E. I hardly ever feel full); 3. Food tastes (A. Very bad/B. Bad/C. Average/D. Good/E. Very good); 4. Normally I eat (A. Less than one meal a day/B. One meal a day/C. Two meals a day/D. Three meals a day/E. More than three meals a day). For each question, the answer scales were $A=1, B=2, C=$ $3, D=4$, and $E=5$. Participants selected the correct answer that best fit their current status. The total SNAQ scores range from 4 to 20 . Patients with a SNAQ score $<14$ were identified to have a poor appetite 11.

The SNAQ was structured using a mobile app called "Sojump", and the QR code of the questionnaire was pushed to patients via WeChat. Participants were asked to complete the questionnaire on the third day of hospital admission. After 7 days of intervention, the participants' appetite was assessed using the SNAQ again. The whole survey process was conducted by the front-line nurses who were providing care for the COVID-19 patients.

\subsection{Intervention}

The procedure of abdominal massage adopted in this study is in accordance with a previous study 12 . The abdominal massage movement pattern consisted of four basic strokes (stroking, effleurage, kneading and vibration) that were applied in a clockwise direction over the intestines through the abdominal wall. 
ST-36 (Zusanli) is bilaterally located approximately four fingers breadth from the lower border of the patella and lateral to the tibia. After locating the ST-36 (Zusanli) point, the patient was asked to press on it once per second with their thumb five times and then to rest for two seconds. The cycle was repeated for three minutes. A 1-1.5 cm depth should be reached for each press. The procedure was repeated on the contralateral leg.

\subsection{Validity and reliability}

The patients who met our inclusion criteria were randomly divided into the experimental group or control group based on a computer-generated list.

To enhance the validity of the intervention, in the experimental group, a 5-minute video-guided training session that was recorded by a nutritional specialist nurse was pushed to participants via WeChat. The patients were instructed to perform abdominal massage and ST-36 (Zusanli) acupressure during the first three days after hospitalization by front-line nurses. Following the training, the participants applied abdominal massage and ST-36 (Zusanli) acupressure by themselves three times a day for one week. In the control group, the patients received regular treatment and nursing.

\subsection{Data analysis}

The statistical analysis was completed using SPSS statistical software (SPSS 20.0 package). Continuous data are reported by mean \pm SD. Categorical variables are shown as absolute numbers and percentages $(n, \%)$. The Wilcoxon marked rank test was used to analyse the numerical and percentage distribution data. $p<0.05$ was defined as the limit of significance.

\section{Results}

\subsection{Demographic and clinical characteristics}

A total of 110 patients were enrolled for final analysis in this study. Basic demographic information was collected at the beginning of hospitalization. The distribution of the participants in terms of their demographic characteristics is shown in Table 1. The mean age of the participants was $46.9 \pm 12.45$ years, $48.2 \%(n=53)$ were female, $75.5 \%(n=83)$ were married, $34.5 \%(n=38)$ were university graduates, and $27.3 \%(n=30)$ had fever symptoms. 
Table 1

Characteristics of the study population.

\begin{tabular}{|ll|}
\hline Variables & $\mathbf{n}(\%)$ \\
\hline Mean of ages & $46.9 \pm 12.45$ \\
\hline Gender & \\
\hline Male & $57(51.8 \%)$ \\
\hline Female & $53(48.2 \%)$ \\
\hline Education status & \\
\hline University graduate & $38(34.5 \%)$ \\
\hline Without university degree & $72(65.5 \%)$ \\
\hline Marriage status & \\
\hline Single & $27(24.5 \%)$ \\
\hline Married & $83(75.5 \%)$ \\
\hline Fever or not & \\
\hline Fever & $30(27.3 \%)$ \\
\hline Not fever & $80(72.7 \%)$ \\
\hline
\end{tabular}

The SNAQ scores of the participants were collected on the third day of hospitalization as a baseline. As shown in Table 2, 39.1\% $(n=43)$ of patients were considered to have a poor appetite according to the SNAQ scores (score < 14). First, we analysed the correlation between the baseline SNAQ scores and sex, age and fever. Our data suggested that older patients and patients with fever were more likely to have a poor appetite $(p<0.05)$. No statistically positive association was observed between a poor appetite and sex. 
Table 2

Related factors of SNAQ scores.

\begin{tabular}{|c|c|c|c|c|}
\hline \multirow[t]{2}{*}{ Variables } & \multicolumn{4}{|l|}{ SNAQ scores } \\
\hline & $<14(\mathrm{n}=43)$ & $\geq 14(\mathrm{n}=67)$ & & \\
\hline \multirow[t]{2}{*}{ Age } & $\overline{\mathrm{x}} \pm S D$ & $\overline{\mathrm{X}} \pm S D$ & $\mathbf{t}$ & $p$ \\
\hline & $50.37 \pm 11.91$ & $44.67 \pm 12.37$ & 2.392 & 0.0185 \\
\hline Gender & $\mathrm{n}(\%)$ & $\mathrm{n}(\%)$ & $x^{2}$ & p \\
\hline Male & $19(44.2)$ & $38(56.7)$ & 1.647 & 0.1994 \\
\hline Female & $24(55.8)$ & $29(43.3)$ & & \\
\hline Fever or not & n (\%) & $\mathrm{n}(\%)$ & & \\
\hline Yes & $18(41.9)$ & $12(17.9)$ & 7.574 & 0.0059 \\
\hline No & $25(58.1)$ & $55(82.1)$ & & \\
\hline
\end{tabular}

The patients' characteristics are summarized in Table 3. All of the variables were analysed with an independent t-test or the chi-square test, and no differences were observed between the two groups. 
Table 3

Comparison of characteristics between the experimental and the control group.

\begin{tabular}{|c|c|c|c|c|c|c|}
\hline \multirow{2}{*}{$\begin{array}{l}\text { Variables } \\
\text { Age }\end{array}$} & \multicolumn{2}{|c|}{$\begin{array}{l}\text { Experimental } \\
(n=55)\end{array}$} & \multicolumn{4}{|l|}{$\begin{array}{l}\text { Control } \\
(n=55)\end{array}$} \\
\hline & Min-Max & $\overline{\mathrm{x}} \pm S D$ & Min-Max & $\overline{\mathrm{x}} \pm S D$ & $t$ & $P$ \\
\hline & $22-67$ & $45.67 \pm 13.27$ & $27-66$ & $48.13 \pm 11.57$ & 1.04 & 0.30 \\
\hline & $\mathrm{n}$ & $\%$ & $\mathrm{n}$ & $\%$ & $x^{2}$ & $P$ \\
\hline \multicolumn{7}{|l|}{ Gender } \\
\hline Male & 27 & $49.1 \%$ & 30 & $54.5 \%$ & 0.33 & 0.57 \\
\hline Female & 28 & $50.9 \%$ & 25 & $45.5 \%$ & & \\
\hline \multicolumn{7}{|l|}{ Education status } \\
\hline University & 16 & $29.1 \%$ & 22 & $40 \%$ & 1.45 & 0.23 \\
\hline Under university & 39 & $70.9 \%$ & 33 & $60 \%$ & & \\
\hline \multicolumn{7}{|l|}{ Marriage status } \\
\hline Single & 12 & $21.8 \%$ & 15 & $27.3 \%$ & 0.44 & 0.51 \\
\hline Married & 43 & $78.2 \%$ & 40 & $72.7 \%$ & & \\
\hline \multicolumn{7}{|l|}{ Fever or not } \\
\hline Fever & 16 & $29.1 \%$ & 14 & $25.5 \%$ & 0.18 & 0.66 \\
\hline Not fever & 39 & $70.9 \%$ & 41 & $74.5 \%$ & & \\
\hline \multicolumn{7}{|l|}{ SNAQ score } \\
\hline$<14$ & 23 & $41.8 \%$ & 20 & $36.4 \%$ & 0.34 & 0.56 \\
\hline$\geq 14$ & 32 & $58.2 \%$ & 35 & $63.6 \%$ & & \\
\hline
\end{tabular}

\subsection{Effect of abdominal massage and ST-36 (Zusanli) acupressure on improving the appetite of COVID-19 patients}

To assess the effects of abdominal massage and ST-36 (Zusanli) acupressure, we collected the SNAQ scores again after 7 days of intervention. As shown in Tables 4 and 5 , in the experimental group, the SNAQ scores significantly increased $(14.07 \pm 2.46$ vs $15.55 \pm 1.77, p=0.0004)$, and the proportion of patients with a poor appetite statistically decreased after the intervention $(41.8 \%$ vs $10.9 \%, p=0.0002)$, while no differences were noted in the control group between baseline and postintervention. Furthermore, 
the SNAQ scores postintervention in the experimental group were significantly higher than those in the control group $(15.55 \pm 1.77$ vs $14.42 \pm 2.59, p=0.0087)$. Importantly, compared with the control group, the proportion of patients with a poor appetite obviously decreased in the experimental group after 7 days of intervention $(27.3 \%$ vs $10.9 \%, p=0.029)$. Our results suggest that abdominal massage and ST-36 (Zusanli) acupressure are beneficial for improving the appetite of mild COVID-19 patients.

Table 4

SNAQ score.

\begin{tabular}{|lllll|}
\hline Groups & SNAQ score & & $\mathbf{t}$ & $\mathbf{p}$ \\
\hline & Pre-test & Post-test & & \\
& $\overline{\mathrm{x}} \pm$ SD & $\overline{\mathrm{x}} \pm$ SD & & \\
\hline Experimental & $14.07 \pm 2.46$ & $15.55 \pm 1.77$ & 3.62 & 0.0004 \\
\hline Control & $14.04 \pm 2.58$ & $14.42 \pm 2.59$ & 0.77 & 0.4425 \\
$\mathbf{t}$ & 0.06 & 2.671 & & \\
$\mathbf{p}$ & 0.9504 & 0.0087 & & \\
\hline
\end{tabular}

Table 5

Proportion of patients with poor appetite.

\begin{tabular}{|c|c|c|c|c|}
\hline \multirow[t]{2}{*}{ Groups } & \multicolumn{2}{|c|}{ SNAQ score $<14$} & \multirow[t]{2}{*}{$x^{2}$} & \multirow[t]{2}{*}{ p } \\
\hline & Pre-test & Post-test & & \\
\hline & $\mathrm{n}(\%)$ & $\mathrm{n}(\%)$ & & \\
\hline Experimental & $23(41.8)$ & $6(10.9)$ & 13.5 & 0.0002 \\
\hline Control & $20(36.4)$ & 15 (27.3) & 1.05 & 0.3067 \\
\hline$x^{2}$ & 0.3436 & 4.767 & & \\
\hline p & 0.5577 & 0.029 & & \\
\hline
\end{tabular}

\section{Discussion}

Since SARS-CoV-2 possesses powerful pathogenicity and transmissibility, the COVID-19 outbreak has become a global public health problem. It is known that the immune response is weakened by inadequate nutrition ${ }^{13}$; thus, adequate nutrition intake deserves attention during COVID-19 treatment. Patients who are confirmed to be infected with the novel coronavirus should be isolated, leading to mental anxiety and poor appetite. In this study, we investigated appetite in 110 COVID-19 patients who were cared for in a 
sport centre makeshift hospital and assessed the effect of abdominal massage and ST-36 (Zusanli) acupressure on improving their appetite. Our results indicated that the proportion of COVID-19 patients with a poor appetite was at a relatively high level compared with other studies in which appetite was also evaluated using SNAQ ${ }^{14-16}$. Our intervention effectively improved their appetite during isolation treatment. To the best of our knowledge, this is the first study focusing on the appetite of COVID-19 patients, especially in the context of makeshift hospitals.

There are a few instruments for assessing appetite in different studies, such as The Functional Assessment of Anorexia/Cachexia Therapy (FACCT), Memorial Symptom Assessment Scale (MSAS) and Appetite, Hunger Feelings, and Sensory Perception (AHSP). However, it takes a long time to complete these measurements, and some of them are designed for cancer patients. In a makeshift hospital, hundreds of patients would be hospitalized in a short period, and complex questionnaires are not applicable. The SNAQ is a brief test that was developed in 2005 by the Council for Nutritional Strategies in Long Term Care in institutionalized and community-dwelling adults ${ }^{17}$. It has been applied in various diseases to estimate the loss of appetite and the risk of weight loss ${ }^{18}$. A recent study reported the usefulness of SNAQ in Chinese patients with liver cirrhosis ${ }^{11}$.

In this study, we evaluated the appetite of COVID-19 patients using a mobile app-based SNAQ. We found that a considerable proportion of COVID-19 patients treated in makeshift hospitals suffered from a poor appetite. The reasons may be as follows: 1 . COVID-19 is not only a respiratory symptom but also affects the digestive system; 2. Psychological problems such as anxiety and fear may result in worse gastrointestinal function; 3 . Reduced access to the habitual diet and a lack of exercise could be considered objective factors.

This inspired us to consider how to improve the appetite of these patients. Abdominal massage and ST36 (Zusanli) acupressure are both nonpharmacological and noninvasive methods that can increase gastric and bowel motility ${ }^{9,11}$. Moreover, these two methods are easy to master and conduct by patients themselves. To standardize the intervention process, an instructional video was recorded by a nutritional specialist nurse. In addition to training with the video, participants were guided by front-line nurses before the beginning of the formal intervention. Encouragingly, most participants confirmed the effectiveness of the video. Most importantly, our intervention played a beneficial role in improving their appetite.

Our study has a number of limitations. First, it was a single-centre study, and there might be some bias in patients' selection. Thus, further multi-centre studies will be helpful. In addition, psychological changes during treatment might influence patients' digestive function, such as appetite. There was a psychotherapist on our treatment team, and psychological guidance was provided to all of the patients. However, psychological factors were not considered in our data.

\section{Conclusion}


In conclusion, our results suggest that COVID-19 patients treated in makeshift hospitals are likely to suffer from a poor appetite for various reasons. Abdominal massage and ST-36 (Zusanli) acupressure are applicable and effective methods for improving appetite. Since COVID-19 has become a global pandemic, we hope that our study may provide some experiences for treating and caring for COVID-19 patients.

\section{Declarations}

\section{Contribution of authors}

Fang Li and Lei Wang: Conceptualization, Data curation, Formal analysis. Lei Wang: Software, Visualization, Writing, Investigation, Project administration. Hui Yuan: Data curation, Methodology, Validation, Writing-review \& editing. Jifang Peng, Hairong Liu, Yan Jia, Cui Yao and Yufan Lin: Formal analysis, Investigation, Project administration.

\section{Conflicts of Interest}

The authors declare no conflict of interest.

\section{Acknowledgements}

We would like to express our appreciation to all participants.

\section{Funding Statement}

This research received no external funding.

\section{Availability of data and materials}

The datasets used or analyzed in the current study are available from the corresponding author on reasonable request.

\section{Ethics approval and consent to participate}

This study was approved by the Ethics Committee of the First Affiliated Hospital of Nanjing Medical University (approval number: 2020-SR-149) on 16 February 2020. All participants were provided written informed consent before enrolment. This study is designed in accordance with the principles of the Declaration of Helsinki.

\section{Consent for publication}

Not applicable.

\section{References}


1. Zhou F, Yu T, Du R, Fan G, Liu Y, Liu Z, et al. Clinical course and risk factors for mortality of adult inpatients with COVID-19 in Wuhan, China: a retrospective cohort study. Lancet. 2020. 10.1016/s0140-6736(20)30566-3.

2. Remuzzi A, Remuzzi G COVID-19 and Italy: what next? Lancet 2020. 10.1016/s0140-6736(20)306279.

3. Laviano A, Koverech A, Zanetti M. Nutrition support in the time of SARS-CoV-2 (COVID-19). Nutrition. 2020 Jun;74:110834. doi:10.1016/j.nut.2020.110834.

4. Huang C, Wang Y, Li X, Ren L, Zhao J, Hu Y, et al. Clinical features of patients infected with 2019 novel coronavirus in Wuhan, China. Lancet. 2020;395:497-506. 10.1016/s0140-6736(20)30183-5.

5. Li T, Zhang Y, Gong C, Wang J, Liu B, Shi L, et al. Prevalence of malnutrition and analysis of related factors in elderly patients with COVID-19 in Wuhan, China. Eur J Clin Nutr 2020; 1-5. 10.1038/s41430-020-0642-3.

6. Birimoglu Okuyan C, Bilgili N. Effect of abdominal massage on constipation and quality of life in older adults: A randomized controlled trial. Complement Ther Med. 2019;47:102219. 10.1016/j.ctim.2019.102219.

7. McClurg D, Hagen S, Jamieson K, Dickinson L, Paul L, Cunnington A. Abdominal massage for the alleviation of symptoms of constipation in people with Parkinson's: a randomised controlled pilot study. Age Ageing. 2016;45:299-303. 10.1093/ageing/afw005.

8. Khanghah AG, Rizi MS, Nabi BN, Adib M, Leili EK. N. Effects of Acupressure on Fatigue in Patients with Cancer Who Underwent Chemotherapy. J Acupunct Meridian Stud. 2019;12:103-10. 10.1016/j.jams.2019.07.003.

9. Chao HL, Miao SJ, Liu PF, Lee HH, Chen YM, Yao CT, et al. The beneficial effect of ST-36 (Zusanli) acupressure on postoperative gastrointestinal function in patients with colorectal cancer. Oncol Nurs Forum. 2013;40:E61-8. 10.1188/13.Onf.E61-e68.

10. Hsiung WT, Chang YC, Yeh ML, Chang YH. Acupressure improves the postoperative comfort of gastric cancer patients: A randomised controlled trial. Complement Ther Med. 2015;23:339-46. 10.1016/j.ctim.2015.03.010.

11. Wang T, Shen J. Usefulness of Simplified Nutritional Appetite Questionnaire (SNAQ) in Appetite Assessment in Elder Patients with Liver Cirrhosis. J Nutr Health Aging. 2018;22:911-5. 10.1007/s12603-018-1086-5.

12. Uysal N, Eşer I, Akpinar $\mathrm{H}$. The effect of abdominal massage on gastric residual volume: a randomized controlled trial. Gastroenterol Nurs. 2012;35:117-23. 10.1097/SGA.0b013e31824c235a.

13. Sieske L, Janssen G, Babel N, Westhoff TH, Wirth R, Pourhassan M Inflammation, Appetite and Food Intake in Older Hospitalized Patients. Nutrients 2019;11. 10.3390/nu11091986.

14. Mikami Y, Watanabe Y, Edahiro A, Motokawa K, Shirobe M, Yasuda J, et al. Relationship between mortality and Council of Nutrition Appetite Questionnaire scores in Japanese nursing home residents. Nutrition. 2019;57:40-5. 10.1016/j.nut.2018.05.030. 
15. Acar Tek N, Karaçil-Ermumcu M. Determinants of Health Related Quality of Life in Home Dwelling Elderly Population: Appetite and Nutritional Status. J Nutr Health Aging. 2018;22:996-1002. 10.1007/s12603-018-1066-9.

16. Pierik VD, Meskers CGM, Van Ancum JM, Numans ST, Verlaan S, Scheerman K, et al. High risk of malnutrition is associated with low muscle mass in older hospitalized patients - a prospective cohort study. BMC Geriatr. 2017;17:118. 10.1186/s12877-017-0505-5.

17. Wilson MM, Thomas DR, Rubenstein LZ, Chibnall JT, Anderson S, Baxi A, et al. Appetite assessment: simple appetite questionnaire predicts weight loss in community-dwelling adults and nursing home residents. Am J Clin Nutr. 2005;82:1074-81. 10.1093/ajcn/82.5.1074.

18. Cox NJ, Morrison L, Ibrahim K, Robinson SM, Sayer AA, Roberts HC. New horizons in appetite and the anorexia of ageing. Age Ageing. 2020. 10.1093/ageing/afaa014.

19. Yıldırım D, Can G, Köknel Talu G. The efficacy of abdominal massage in managing opioid-induced constipation. Eur J Oncol Nurs. 2019;41:110-9. 10.1016/j.ejon.2019.05.013. 